\title{
artigo
}

Alves, E.G.; Martins, N.C.; Santos, R.M.; Miranda da Silva, S.S.; Ferreira, S.D.R.S.;

Adesão ao tratamento de usuários hipertensos assistidos por uma equipe de estratégia da saúde da familia

\section{Adesão ao tratamento de usuários hipertensos assistidos por uma equipe de estratégía da saúde da família}

\author{
Adherence and therapy of hypertensive users assisted by a family health strategy team \\ Adherencia al tratamiento de usuarios hipertensos asistidos por un equipo de estrategia de salud de la familia
}

\begin{abstract}
RESUMO
Objetivo: 0 presente estudo tem por objetivo relatar às experiências de um projeto de intervenção sobre a sensibilização na adesão ao tratamento de usuários com diagnóstico de Hipertensão arterial sistêmica em uma Unidade Básica de Saúde da Família. Métodos: Trata-se de um relato de experiência, de abordagem qualitativa. Participaram do projeto usuários hipertensos assistidos por uma equipe de estratégia saúde da família no município de Manaus, AM. Resultados: Participaram do grupo operacional 40 usuários. Destes, 19 eram do sexo feminino e 21 do sexo masculino, com idade entre 60 a 79 anos. Quanto à estratificação de risco cardiovascular de Framingham, 30 usuários encontravam-se na categoria de alto risco e 10 em risco moderado. Conclusão: Ações a esta população possibilitam o maior conhecimento sobre a hipertensão, suas complicações e medidas terapêuticas e maior adesão ao tratamento destes pacientes.
\end{abstract}

DESCRITORES: Estratégia saúde da família; hipertensão arterial sistêmica; assistência à saúde; educação em saúde.

\section{ABSTRACT}

Objective: The present study aims to report the experiences of an intervention project on sensitization in adhering to the treatment of users diagnosed with systemic arterial hypertension in a Basic Family Health Unit. Methods: Es un relato de experiencia, con enfoque cualitativo. El proyecto involucró a usuarios hipertensos que estaban siendo monitoreados en un hospital asistido por un equipo de estrategia de salud familiar en la ciudad de Manaus, AM. Results: 40 users participated in the operational group. Of these, 19 were female and 21 male, aged 60 to 79 years. As for the Framingham cardiovascular risk stratification, 30 users were in the high risk category and 10 at moderate risk. Conclusion: Actions for this population enable an improvement in knowledge about hypertension, its complications and treatment measures and, consequently, greater adherence to treatment. DESCRIPTORS: Family health strategy; systemic arterial hypertension; health care; health education.

\section{RESUMEN}

Objetivo: El presente estudio tiene como objetivo relatar las experiencias de un proyecto de intervención sobre sensibilización en la adherencia al tratamiento de usuarios diagnosticados de hipertensión arterial sistémica en una Unidad Básica de Salud de la Familia. Métodos: Se trata de un relato de experiencia, con enfoque cualitativo. Los usuarios hipertensos que participan en una estrategia de salud familiar participaron del proyecto en la ciudad de Manaus, AM. Resultados: 40 usuarios participaron en el grupo operativo. De ellos, 19 eran mujeres y 21 hombres, de entre 60 y 79 años. En cuanto a la estratificación de riesgo cardiovascular de Framingham, 30 usuarios estaban en la categoría de alto riesgo y 10 en riesgo moderado. Conclusión: Las acciones dirigidas a esta población permiten mejorar el conocimiento sobre la hipertensión, sus complicaciones y medidas de tratamiento y, en consecuencia, una mayor adherencia al tratamiento.

DESCRIPTORES: Estrategia de Salud de la Familia; Hipertensión Arterial Sistémica; Atención en Salud; Educación en Salud.

RECEBIDO EM: 15/10/2020 APROVADO EM: 12/01/2021

\section{Erika Gomes Alves}

Fisioterapeuta. Escola de Saúde Pública de Manaus - ESAP.

ORCID: 0000-0002-4565-4775

\section{Nádia Cardoso Martins}

Enfermeiro. Escola de Saúde Pública de Manaus - ESAP.

ORCID: 0000-0002-1107-4270 


\section{Rednaj Mota Santos}

Enfermeiro. Escola de Saúde Pública de Manaus - ESAP.

ORCID: 0000-0001-6558-9758

\section{Sabrina Sonra Miranda da Silva}

Enfermeiro. Escola de Saúde Pública de Manaus - ESAP.

ORCID: 0000-0003-0141-4769

\section{Sandra Deyse Rodrigues Souza Ferreira}

Enfermeiro. Escola de Saúde Pública de Manaus - ESAP.

ORCID: 0000-0002-3946-6743

\section{INTRODUÇÃO}

A s Doenças Crônicas Não Transmissíveis (DCNT) constituem um dos maiores problemas de saúde pública no Brasil e no mundo. A hipertensão arterial sistêmica (HAS) está entre os cinco principais agravos que quando não controlada, ocasiona uma série de complicações, como doenças cardiovasculares e renais, aumentando o número de internaçôes hospitalares e gerando altos custos para o indivíduo e a sociedade ${ }^{1}$.

Define-se HAS como uma condição clínica multifatorial caracterizada por elevação e sustentação dos níveis pressóricos $\geq 140$ e/ou $90 \mathrm{mmHg}$ associada frequentemente a distúrbios metabólicos, alterações funcionais e/ou estruturais de órgãos-alvo ${ }^{2}$.

A HAS possui três características importantes, a primeira é a prevalência: responsável por uma parcela significativa das consultas da rede básica; a segunda é a transcendência, considerada um dos principais fatores de risco associado ao acidente vascular encefálico, infarto agudo do miocárdio (IAM). E por último, temos a vulnerabilidade, facilmente tratável e controlável no âmbito da Atenção Primária em Saúde (APS), uma vez que 50 a $80 \%$ dos casos se resolve na rede básica ${ }^{3}$.

A APS é a porta de entrada de usuários na prevenção, controle e tratamento de doenças crônicas como a HAS, sendo de responsabilidade favorecer o vínculo com a comunidade e a clientela da área adstrita, trabalhando fatores que precisam ser adequadamente abordados e controlados, levando em conta a diversidade racial, cultu- ral, religiosa e os fatores sociais envolvidos4.

A equipe de APS tem importância primordial nas estratégias de prevenção, diagnóstico, monitoramento e controle da hipertensão arterial. Devem também, ter sempre em foco o princípio fundamental da prática centrada na pessoa e, consequentemente, envolver usuários e cuidadores, em nível individual e coletivo, na definição e implementação de estratégias de controle à hipertensão ${ }^{4}$.

O tratamento para HAS caracteriza-se como não medicamentoso e medicamentoso, ambos, individual ou combinado, visam à redução da morbimortalidade cardiovascular. Pessoas com hipertensão leve podem ser tratadas sem o uso de medicamento, através da modificação no estilo de vida, alimentação adequada, sobretudo quanto ao consumo de sal e ao controle do peso, a prática de atividade física, o abandono do tabagismo e a redução do uso excessivo de álcool ${ }^{4,5}$.

A terapia medicamentosa tem como base o uso de fármacos diuréticos, betabloqueadores, inibidores da enzima conversora da angiotensina, bloqueadores do receptor AT1 da angiotensina (BRA II), e antagonistas dos canais de cálcio, de uso combinado ou individual, reduzindo a pressão arterial e os eventos cardiovasculares fatais e não fatais ${ }^{6}$.

$\mathrm{Na}$ rotina das unidades de saúde, o usuário portador de HAS é um constante frequentador em busca da renovação de receitas, consulta de acompanhamento, verificação da pressão e atendimento para a agudizarão de sua condição crônica, entre outras, porém, muitas dessas visitas podem estar relacionadas a não adesão $\mathrm{a}$ um tratamento medicamentoso prescrito, situação essa que pode ocorrer por vários fatores $^{7,8}$.

A adesão ao tratamento farmacológico da HAS é considerada uma das etapas essenciais para a garantia do seu sucesso. Para tanto, o conhecimento da doença, suas complicações e necessidade de mudanças em relação ao estilo de vida, requer do paciente, além da motivação, orientação contínua e de modo compartilhado.

A não adesão ao tratamento resulta $\mathrm{em}$ agravos no processo patológico, aumento no número de morbidades e internaçôes hospitalares. Deste modo, medidas que estimulem a melhor adesão terapêutica são importantes, através do fortalecimento do vinculo entre profissionais e usuários, além do desenvolvimento de ações de educação, prevenção e promoção à saúde.

Este trabalho tem por objetivo relatar às experiências de um projeto de intervenção (PI) sobre a sensibilização na adesão ao tratamento de usuários com diagnóstico de Hipertensão arterial sistêmica em uma Unidade Básica de Saúde da Família.

\section{MÉTODO}

Trata-se de um relato de experiência, de abordagem qualitativa e de caráter descritivo, de um projeto de intervenção(PI) realizado em uma Unidade Básica de Saúde da Família, na Zona Leste, no período de janeiro a fevereiro de 2020, na cidade de Manaus, Amazonas, . Este PI faz parte do trabalho de conclusão do curso de Especialização em Saúde Pública, com ênfase em Estratégia Saúde da Família realizado pela Escola de Saúde Pública de Manaus (ESAP) em parceria com a Universidade do Estado do Amazonas (UEA). 
A unidade de saúde possui sete microáreas inseridas em seu território sendo composta por (1) médico, (1) enfermeira, (2) técnicos de enfermagem e (7) agentes comunitários de saúde - ACS's.

As atividades foram desenvolvidas através da organização de um grupo operacional de usuários hipertensos $(\mathrm{n}=40)$. As ações realizadas foram: consulta domiciliar, classificação do risco cardiovascular, levantamento epidemiológico e grupo educativo, tendo como enfoque principal o nível de entendimento das informações obtidas, as relações dentro do grupo e os sentimentos e expectativas relatados.

Participara do grupo operacional usuários assistidos pela ESF que atenderam os seguintes critérios: : diagnóstico de HAS, estar regularmente cadastrado no sistema de informação e-SUS (completo), exames laboratoriais (lipidograma, uréia e creatina) atualizados (até 12 meses), classificado com risco cardiovascular moderado ou alto a partir da estratificação de Framingham. Foram inelegíveis ao projeto de intervenção: usuários com diagnóstico equivocado e tardio de HAS, pacientes que recusaram à consulta domiciliar e que não se encontravam em suas residências.

A adesão ao tratamento dos hipertensos integrantes do grupo operacional foi determinada por meio do autorrelato, durante entrevista, garantindo total sigilo e respeito ao usuário.

Quanto à classificação de risco cardiovascular utilizou-se o escore de Framingham e do aplicativo cardiac risck calculator, sendo o paciente classificado nas seguintes categorias: baixo, intermediário/moderado e alto risco. Este aplicativo calcula o risco cardiovascular baseado em alguns parâmetros de entrada de usuários e na equação de Framingham.

\section{A execução do PI dividiu-se em três etapas:}

1 etapa; capacitação da equipe, rastreamento dos usuários. Nesta fase foi realizada a educação permanente com os profissionais que integram a ESF através da roda de conversa, foram abordados os temas: hipertensão arterial sistêmica, adesão me- dicamentosa e não medicamentosa, atendimento domiciliar e educação em saúde. $\mathrm{O}$ objetivo da atividade era identificar o conhecimento prévio dos ACS's sobre a proposta da aplicação do projeto de intervenção e realizar o convite para os mesmos colaborarem diretamente no projeto.

As atividades foram desenvolvidas através da organização de um grupo operacional de usuários hipertensos ( $n=40)$. As ações realizadas foram: consulta domiciliar, classificação do risco cardiovascular,

levantamento epidemiológico e grupo educativo, tendo como enfoque principal o nível de entendimento das informações obtidas, as relações dentro do grupo e os sentimentos e expectativas relatados.
2 etapa; visita domiciliar, adesão ao tratamento e classificação de risco cardiovascular. A adesão ao tratamento foi avaliada a partir do autorrelato durante as visitas domiciliares. Estas ocorreram, uma vez por semana, sendo realizada pelos enfermeiros especializandos e ACSs, organizados em dois grupos. Cada grupo era composto por um ACS e dois enfermeiros especializandos. No decorrer das atividades de atendimento domiciliar, foram realizadas as avaliações antropométricas, verificação dos níveis pressóricos, atendimento clínico de Enfermagem, avalição multidimensional e convite para as atividades de educação em saúde.

3 etapa; ações de educação em saúde em grupo operacional (2 encontros). Os temas abordados nas atividades em grupo foram: DCNT; tratamento medicamentoso e não medicamentoso; alimentação saudável e importância da prática de atividades físicas. Nesta etapa foram executadas as atividades de educação em saúde para o grupo de pacientes com diagnóstico de HAS. Foram realizados dois encontros, antes da atividade proposta foram verificados os níveis pressóricos, peso, altura e circunferência abdominal de todos os participantes. Os dados foram registrados e utilizados como base para orientação terapêutica durante a consulta no domicilio

A primeira ação de educação em saúde foi realizada no período matutino, com duração de duas horas. A atividade foi mediada pelas profissionais farmacêutica e uma nutricionista, ambas profissionais do Distrito de Saúde Leste - DISAL. Utilizou-se como metodologia a roda de conversa, abordando os seguintes temas: medicamentos usados para HAS, adesão medicamentosa e não medicamentosa, hábitos de vida e alimentação saudável

O segundo encontro foi realizado no período vespertino. Estiveram presentes para mediar à ação os profissionais: odontologista e os enfermeiros especializandos. Foram abordados na roda de conversa os seguintes temas: higiene oral, cuidados com os dentes e próteses dentárias, desafios quanto ao acesso ambu- 
latorial, acesso a farmácia e aos medicamentos prescritos pelo médico e cuidado sistematizado dos profissionais enfermeiros e médicos.

Os dados foram organizados e posteriormente analisados a partir da experiência dos usuários quanto à doença e as medidas de tratamento. Os dados foram digitados em um banco de dados do programa Microsoft Excel 2019, as variáveis foram apresentadas de forma descritiva através de tabelas.

Este estudo teve aprovação do Comitê de Ética em Pesquisa em Seres Humanos da Universidade Federal do Amazonas - UFAM, com CAAE $\mathrm{n}^{\circ}$ 20092919.5.00005020.

\section{RESULTADOS}

Do total de usuários cadastrados (3.266), cento e sessenta e quatro (164) eram hipertensos e setenta e dois (72) eram hipertensos e diabéticos. A amostra foi constituída por 40 hipertensos, dos quais (21) eram homens. Houve predomínio de participantes com idade entre 60 a 70 anos. No que tange a escolaridade, a maioria relatou ter o ensino fundamental completo (19). Quanto ao risco cardiovascular a partir do escore de Framingham, observamos que (30) usuários apresentaram alto risco e (10) apresentaram risco moderado. A seguir apresentamos a caracterização dos participantes (tabela 1).

Quanto à estratificação de risco cardiovascular de Framingham, 30 usuários encontravam-se na categoria de alto risco e $10 \mathrm{em}$ risco moderado.

Em relação à adesão terapêutica, foram identificados 2 grupos de usuários: que aderiram parcialmente ou não aderiram ao tratamento proposto. Entre as principais dificuldades relatadas quanto à adesão ao tratamento por estes indivíduos, estão: a realização de atividades domésticas, cuidar de familiares, distanciamentos dos filhos, renda baixa, desconhecimento dos programas governamentais, uso incorreto das medicações anti-hipertensivas, dificuldade de compreensão sobre a prescrição medicamentosa, aprazamentos, reações adversas, uso concomitantes com outros medicamentos e fitoterápicos.

\section{DISCUSSÃO}

A adesão ao tratamento é um fenômeno que pode envolver múltiplos fatores relacionados ao paciente, prescrição terapêutica, patologia, acesso aos serviços de saúde, bem como com o meio social e cultural do usuário e sua família. A partir da identificação destes fatores é possível o estabelecimento de medidas de intervenção.

Tabela 1. Características dos usuários da Unidade Básica de Saúde participantes do Projeto de Intervenção. Manaus - AM, 2020.

$$
\text { VARIÁVEL N (40) }
$$

\section{Sexo}

Masculino

Feminino

Idade

60 a 70

Escolaridade

Ensino Fundamental

Ensino Médio

Analfabeto

4

Risco cadiovascular de Framingham

Alto Risco

Risco Moderado
O estabelecimento de um bom relacionamento entre profissionais da saúde e pacientes pode melhorar a adesão ao tratamento. $\mathrm{O}$ enfoque diferenciado dos diversos profissionais que compõem a ESF é fundamental para uma melhor abordagem das DCNT. Os grandes sistemas de saúde, como o Sistema Único de Saúde (SUS), fundamentam-se em equipes multiprofissionais?.

O manejo de atividades como: roda de conversa, palestras e dinâmicas proporcionam a interatividade entre pacientes e profissionais, possibilitando a troca de saberes e práticas, fazendo com que consigam entender e aprender o processo da doença e consequentemente auxiliar na forma correta do tratamento, condutas e empoderamento para autocuidado a ser realizada no decorrer das complicações ou não da $\mathrm{HAS}^{10}$. A visita domiciliar deve ser utilizada como tecnologia apropriada para a abordagem ao indivíduo em seu aspecto familiar e comunitário, como estratégia importante para a promoção da saúde e como potente indutor da mudança do modelo tecnoassistencial em saúde. Além de representar um espaço de troca de saberes, dispõe de inúmeros potenciais, tais como, o fortalecimento do cuidado familiar; a criação de vínculo entre profissionais com a comunidade. $\mathrm{O}$ domicilio consiste em um espaço de construção coletiva da equipe de saúde e o usuário, favorece o cuidado mais humanizado e facilita a identificar as dificuldades que possibilitam um olhar diferenciado da equipe de saúde para alterações na forma de atuação adaptando-as ao contexto social de cada indivíduo ${ }^{11,12}$.

$\mathrm{O}$ atendimento domiciliar possibilita aos profissionais uma visão holística da vida de cada usuário, beneficiando a implantação de estratégias individuais ou coletivas que possam envolver a família e a comunidade identificando fatores de risco que possam interferir no processo saúde-doença e a realizar intervenções com maiores chances de êxito.

A falta de conhecimento sobre as formas de tratamento e medidas de autocui- 
dado foi constantemente relatada pelos usuários durante as atividades em grupo e visitas domiciliares. Um estudo realizado no Hospital das Clínicas em São Paulo, com pacientes hipertensos, demonstrou que $81 \%$ dos entrevistados desconheciam o tratamento não medicamentoso para $\mathrm{HAS}^{12}$. Cabe aos profissionais da saúde o constante reforço da importância do tratamento não medicamentoso no controle da HAS.

Programas de educação em saúde favorecem a promoção do autocuidado e tornam o usuário mais responsável pelas decisões relacionadas à sua saúde. É importante que essas atividades sejam desenvolvidas de forma multidisciplinar, com atuação de forma integrada na abordagem da avaliação de risco, adoção de medidas de promoção da saúde e atendimento aos usuários dos serviços de acompanhamento ambulatorial ${ }^{13}$.

O programa Estratégia Saúde da Familiar dispõe de um vasto panorama para se trabalhar a promoção da saúde, a prevenção de doenças, o cuidado continuado, e a integração do usuário de acordo com suas características epidemiológicas.
Além disso, devido

à complexidade

da HAS, são

imprescindíveis

abordagens que

envolvam todos

os profissionais da

unidade de saúde,

usuários e seus

familiares, na definição

e pactuação das metas

de acompanhamento a

serem atingidas.

\section{CONCLUSÃO}

O cuidado dos usuários com DCNT representa um desafio para a ESF. Entre estas doenças a HAS é a mais prevalente, sendo considerado um problema de saúde pública.

Além da assistência por parte da equipe é necessário que haja a participação individual na adesão ao tratamento proposto. Ações que estimulem o empoderamento do paciente sobre sua doença são importantes. Além disso, devido à complexidade da HAS, são imprescindíveis abordagens que envolvam todos os profissionais da unidade de saúde, usuários e seus familiares, na definição e pactuação das metas de acompanhamento a serem atingidas

As atividades no grupo de hipertensos possibilitou um espaço de diálogo importante para o planejamento de medidas individuais e coletivas a seus integrantes. É papel da equipe de saúde promover medidas facilitadoras que contribuam para uma maior adesão ao tratamento. A criação do grupo foi considerada fundamental pelos usuários e profissionais da unidade de saúde, estando fundamentado no principio da integralidade do cuidado. -

\section{REFERÊNCIAS}

1. Da Silva B, et al. Hospitalização por agravos da hipertensão arterial em pacientes da atenção primária. v. 26, n. 4, p. 313-317. São Paulo: Acta Paulista de Enfermagem; 2013.

2. Malachias MVB, et al. $7^{\text {a }}$ Diretriz Brasileira de Hipertensão Arterial: Capítulo 3 - Avaliação Clínica e Complementar. Arq. Bras. Cardiol. [Internet]. 2016.

3. Malta DC et al. A Política Nacional de Promoção da Saúde e a agenda da atividade física no contexto do SUS. Epidemiologia. Serv. Saúde; 2009.

4. Brasil, Ministério da Saúde. Secretaria de Atenção à Saúde. Departamento de Atenção Básica. Estratégias para o cuidado da pessoa com doença crônica: hipertensão arterial sistêmica. Brasilia, DF, Ministério da Saúde, 2013.

5. Brandão $A A$, et al. $6^{\text {a }}$ Diretrizes de Monitorização Ambulatorial da Pressão Arterial e $4^{\text {a }}$ Diretrizes de Monitorização Residencial da Pressão Arterial. Arquivos Brasileiros de Cardiologia; 2018.

6. Kohlmann JR, et al. Tratamento medicamentoso. Brazilian Journal of Nephrology, v. 32, p. 29-43, 2010.

7. Brasil, Ministério da Saúde. Secretaria de Atenção à Saúde. Departamento de Atenção Primária em Saúde. Núcleo de Apoio à
Saúde da Familia. Cadernos de Atenção Primária em Saúde n. 39. Brasilia, DF, Ministério da Saúde 2014.

8. Brasil, Ministério da Saúde. Secretaria de Ciência, Tecnologia e Insumos Estratégicos. Departamento de Ciência e Tecnologia. Síntese de evidências para políticas de saúde: adesão ao tratamento medicamentoso por clientes portadores de doenças crônicas. Brasilia, DF, Ministério da Saúde, 2016.

9. De Gusmão, J L et al. Adesão ao tratamento em hipertensão arterial sistólica isolada. Rev Bras Hipertens, v. 16, n. 1, p. 38-43, 2009.

10. Oliveira J, et al. Sociedade Brasileira de Diabetes. Diretrizes da Sociedade Brasileira de Diabetes. São Paulo, SP: Editora Clannad; 2018, 2017.

11. Vasconcelos $M$, et al. Práticas educativas e tecnologias em saúde. Belo Horizonte, MG. Núcleo de Educação em Saúde Coletiva, UFMG; 2014.

12. Drulla, AG, et al. A visita domiciliar como ferramenta ao cuidado familiar. Curitiba, PR. Cogitare Enfermagem; 2009.

13. Mion Jr,et al. Conhecimentos, preferências: o perfil dos hipertensos quanto ao tratamento farmacológico e não farmacológicos. São Paulo, SP. Jornal Brasileiro de Nefrologia; 1995. 